\title{
Is Glycosylated Hemoglobin a good marker for glycaemic control in diabetic patients with G6PDD?
}

Al Osali M., Al Harthi S., Al Qassabi S., and Abd Al Gafar M. Al Nahdha Hospital. Muscat. Oman

Background: Diabetes is a progressive disease that leads to multiple complications. Prospective trials in patients with diabetes $^{1}$ have demonstrated that elevated glycosylated hemoglobin (HbA1C) levels are associated with these complications. Accordingly, The American Diabetes Association recommends $\mathrm{HbA1C}$ as the standard marker of glycemic control $^{2}$. Sometimes there are discrepancies between HbA1C and blood glucose values as in cases with hemoglobinopathies ${ }^{3}$.

Aim: We conducted this study to determine the prevalence of glucose-6-phosphate dehydrogenase deficiency (G6PDD) among diabetic patients and to find out the relation between G6PDD and glycemic control in our population.
Methods: Omani patients who have visited diabetic clinic at Bowsher polyclinic, Muscat, and have type 2 diabetes mellitus (T2DM) were randomly included in the study. Patients who are younger than 40 and/or have other haemoglobinopathies were excluded. Patients were analyzed for G6PDD, HbA1c, Plasma and Capillary Fasting Blood Sugar (FBS), hemoglobin $(\mathrm{Hb})$ and Total Bilirubin (T. Bil) and subjected to demographic and anthropometric features (age, gender, Body Mass Index(BMI). We studied the Prevalence of G6PDD in relation to age, gender and BMI and we compared the levels of HbA1c, Plasma FBS, Capillary FBS, T. Bil and $\mathrm{Hb}$ in normal and G6PDD groups.

\begin{tabular}{|c|c|c|c|}
\hline \multirow{2}{*}{ Variables } & \multicolumn{2}{|c|}{ Mean } & $\begin{array}{c}\text { P } \\
\text { Value }\end{array}$ \\
\cline { 2 - 3 } & D & N & \\
\hline Age & 57.38 & 57.82 & 0.728 \\
\hline BMI & 29.48 & 31.34 & 0.039 \\
\hline Hb & 12.23 & 12.69 & 0.060 \\
\hline Cap FBS & 10.93 & 10.75 & 0.895 \\
\hline Plasma FBS & 11.27 & 10.99 & 0.916 \\
\hline HbA1c & 7.44 & 9.10 & $<0.001$ \\
\hline T. Bilirubin & 13.94 & 9.75 & $<0.001$ \\
\hline
\end{tabular}
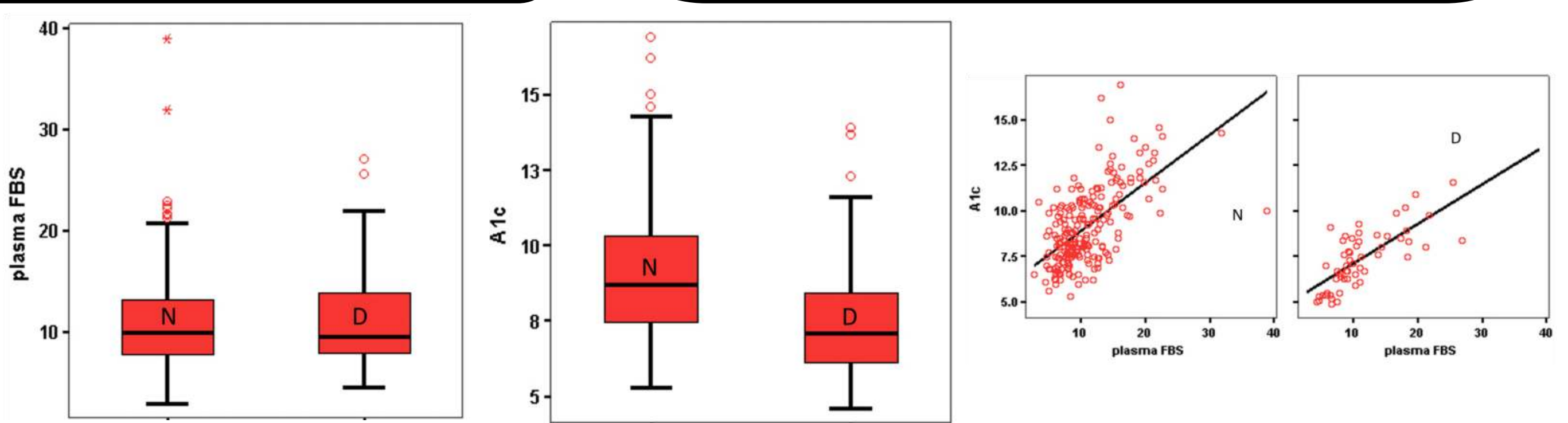

Discussion We found significantly lower HbA1c in D group compared to the $\mathrm{N}$ group [ mean(M) 7.44 vs 9.1 $(\mathrm{P}<0.001)$ ]. We reported also that, FBS whether Capillary [ M 10.93 vs10.75] or Plasma [ M 11.27 vs 10.99] was slightly higher in D group compared to $\mathrm{N}$ group but this was not statistically significant. These figures of FBS indicates poor glycaemic control in both groups. The lower HbAlc in the presence of higher FBS indicating that $\mathrm{HbA1c}$ is a misleading measuring tool of glycaemic control in D group. Despite this significant difference between the $\mathrm{D}$ and $\mathrm{N}$ groups in terms of HbA1c, our study did not show significant difference in $\mathrm{Hb}$ level between the 2 groups [M $12,23$ vs 12.69 respectively $(\mathrm{P}=0.060)]$. Nevertheless, $T$. Bil was slightly but significantly higher in D group compared to the N group, [ M 13.94 vs $9.75(\mathrm{P}<$ 0.001)]. This higher bilirubin without lower Hb levels in the $\mathrm{D}$ group might not reflect actual haemolysis because the averages in both groups are still within normal range in our laboratory $(5-21 \mathrm{umol} / \mathrm{L})$ and we used total rather than indirect bilirubin and in addition, indirect bilirubin itself is not a specific marker for hemolysis because it could be raised by other causes like Gilbert disease $^{4}$. Therefore, the cause of lower HbA1c in the D group could be related to another factor(s) apart from hemolysis, an observation that merits further research
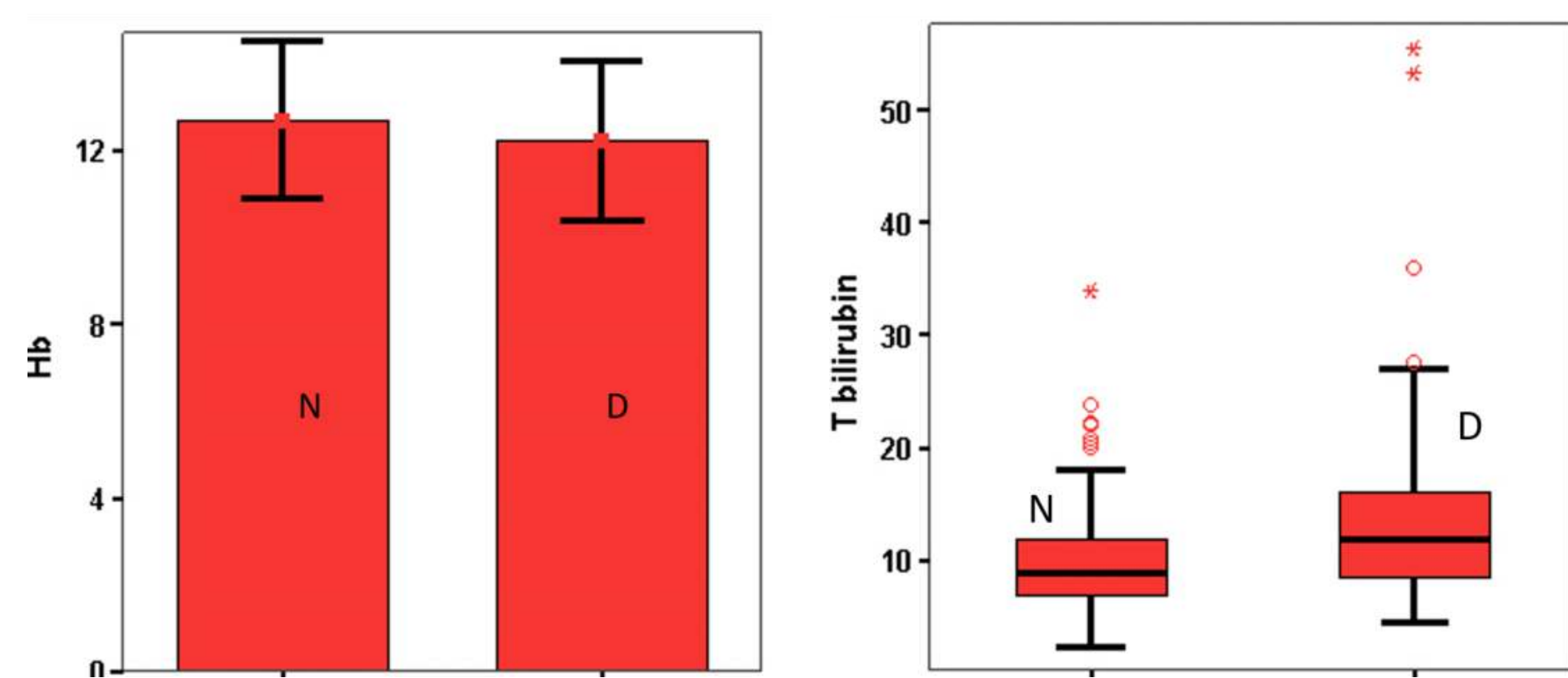

Keywords: G6PDD-T2DM-HbA1c-Hb-T Bilirubin-FBS

Acknowledgements: Our gratitude to Mrs. Aliya Abdalaziz al Balushi at Bowsher polyclinic for her contribution in the study.
Results: 365 Omani patients (190 Men, and 175 Women) who have T2DM and are 40 years old or above are included. Among them, $90(24.7 \%)$ have G6PDD (D group) and $275(75.3 \%)$ have normal G6PD levels ( $\mathrm{N}$ group). In the $\mathrm{D}$ Group, 57 are men (63.3\%) and 33 are women $(36.7 \%)$ [P=0.014]. The differences between the 2 groups about variables are seen in the table and figures.

Conclusion: G6PDD is common among diabetic Omani population. HbAlc is not a good marker for glycemic control in patients with G6PDD necessitating screening diabetic patients with lower HbA1c for G6PDD. The cause of lower $\mathrm{HbAlc}$ in the deficient group could be related to another factor(s) apart from hemolysis, an observation that merits further research. We recommend other tools as fructosamine level as a marker for glycaemic control in diabetic patients with G6PDD.
References

1. doi: $10.1046 / j .1365-2125.1999 .00092 . x$

2. ADA: Standards of medical care in diabetes. Diabetes Care. 2007;30: S4-S41.

. Reynolds TM et al. HbA1c monitoring. BMJ. 2006; 333:586-8.

4. Schick P. Hemolytic Anemia. Medscape. Accessed in 27 Oct. 2017.
Corresponding Author: Dr. Magdi Al Osali

E mail: mag_alosali@yahoo.com.

The Authors have no conflict of interest 